\title{
SHALLOW WATER TOMOGRAPHY IN A HIGHLY VARIABLE SCENARIO
}

\author{
CRISTIANO SOARES AND SÉRGIO M.JESUS \\ SiPLAB-FCT, Universidade do Algarve \\ Campus de Gambelas, PT-8005-139 Faro, Portugal \\ E-mail: \{csoares,sjesus\}@ualg.pt \\ EMANUEL COELHO \\ NATO Undersea Research Centre \\ Viale San Bartolomeo 400, IT-19138 La Spezia, Italy \\ E-mail: coelho@saclantc.nato.int
}

In October 2000, SiPLAB and the Instituto Hidrográfico (IH - PN) conducted the INTIFANTE'00 sea trial in a shallow area off the Peninsula of Tróia, approximately $50 \mathrm{~km}$ south from Lisbon, in Portugal. The experiment itself and results obtained in most of the data set have been reported at various occasions in the last two years. This paper focuses on the data acquired during Event 2, where the acoustic propagation path was approximately range independent and the source ship was held on station at a constant range of $5.8 \mathrm{~km}$ from the vertical line array. Although these conditions were, in general, relatively benign for matched-field tomography, retrieval of water column and bottom parameters over a 14-hour-long recording revealed to be extremely difficult. This paper analysis in detail the characteristics of this data set and determines the causes for the observed inversion difficulties. Is is shown that the causes for the poor performance of the conventional methods are mainly the tide induced spatially correlated noise and the relative source-receiver motion during time averaging. An eigenvalue-based criterion is proposed for detecting optimal averaging time. It is shown that this data selection procedure together with hydrophone normalization and an appropriate objective function provide a better model fit and consistent inversion results and thus a better understanding of the environmental variability.

\section{Introduction}

Ocean Acoustic Tomography (OAT) was initially proposed for deep water regions where the ray approximation was valid and sound speed could be analytically linked to acoustic ray travel-time [1]. Travel-time based tomography turned out to be highly dependent on the ability to separate closely spaced arrivals and on the precise knowledge on the source-receiver relative position at all times. Instead, matched-field tomography (MFT) is based on some sort of correlation of the full pressure field to the signal received at an array of sensors and only requires relative travel times to which an

197

A. Caiti, N.R. Chapman, J.-P. Hermand and S.M. Jesus (eds.), Acoustic Sensing Techniques for the Shallow Water Environment, 197-211.

(C) 2006 Springer. Printed in the Netherlands. 
approximate knowledge of the source-receiver position is sufficient [2]. In most operational shallow water scenarios, only MFT is applicable due to the close arrivals from bottom and surface reflections and to a perpetual source/sensor motion. In October 2000, SiPLAB and the Instituto Hidrografico (IH-PN) conducted the INTIFANTE' O0 sea trial in a shallow water area off the Peninsula of Troía, approximately $50 \mathrm{~km}$ south from Lisbon, in Portugal. The experiment itself and results obtained in part of the data set have been reported during these last two years in various occasions [3], [4], [5], [6]. This paper focuses on the data acquired during Event 2, where the acoustic propagation path was approximately range independent and the source ship was held on station at constant range of $5.8 \mathrm{~km}$ from the vertical line array (VLA). Although these conditions seem ideal for MFT, retrieval of water column and bottom parameters over an 14 hours long recording using a focalization-like procedure turned out to be extremely difficult and resulted in several mismatched parameter estimates.

This paper analyses in detail the characteristics of that data set and the reasons for inversion failure. Separated eigenvalue analysis of the signal plus noise and noise only data sections revealed that signal and noise subspaces are not well separated since the noise showed to have a strong spatial correlation and the signal energy spread over a wide range of eigenvalues. The causes are attributed to both source-receiver motion and tidal current forcing on the VLA. After identifying the causes, several methods to counter these effects are proposed. In particular, a cross-frequency based objective function in conjunction with a proper selection of the data window length, according to the variable source range, and a data normalization for correct initial phase correlation at each frequency contributed for a decrease of the water column and bottom parameter errors. It is shown that this data selection procedure provides a better understanding of the influence of the environment and thus final inversion results that are consistent with independent measurements.

\section{The INTIFANTE'00 sea trial}

The INTIFANTE'00 sea trial was carried out on a shallow water area in the vicinity of Setúbal, situated approximately $50 \mathrm{~km}$ south of Lisbon, during October 2000. This is known to be an area of intense activity in terms of internal tides, internal waves, and river plumes. This sea trial served several purposes: one, among others, was to study the effect of environmental variability in acoustic tomography through long observation times of continuous acoustic transmissions. Event 2 was designed for this purpose, and this study will therefore focus on that data set.

The experimental area was a rectangular box situated in the border of the continental platform with depths varying from 60 to $140 \mathrm{~m}$. Figure 1 shows a bathymetric map of the northern half of the rectangular box. The black thick line represents the track of the research vessel NRP D. Carlos I, towing the sound source, during Event 2 and extending towards the NW of the 16-hydrophone- $4 \mathrm{~m}$ spacing VLA. The bathymetry over this NW leg is considered range-independent with an approximate average depth of $118.7 \mathrm{~m}$.

The research vessel NRP D. Carlos I departed from the VLA location at time 13:30 of Julian day 289 and moved at a constant speed of 1.2 knot along NW leg during 2 hours, as depicted in Figure 1. After an interruption for battery changing, signal transmissions resumed at time 21:00, with the NRP D. Carlos I holding a station at approximately $5.8 \mathrm{~km}$ from the VLA while continuously transmitting acoustic signals until time 11:30 a.m. of Julian day 290. The acoustic aperture of the VLA was between 30 and $90 \mathrm{~m}$. The wave form emitted during Event 2 was a $2 \mathrm{~s}$ duration linear frequency modulated (LFM) sweep in the band 170 to $600 \mathrm{~Hz}$ with a repetition rate of $10 \mathrm{~s}$.

During the INTIMATE'00 sea trial a water column temperature survey using a thermistor chain and XBTs was conducted. The $\mathbf{X}$ signs in figure 1 denote the locations where the XBTs were released every 3 hours during approximately 2 days. These data were used in this study for the computation of the channel characteristic empirical orthogonal functions. 


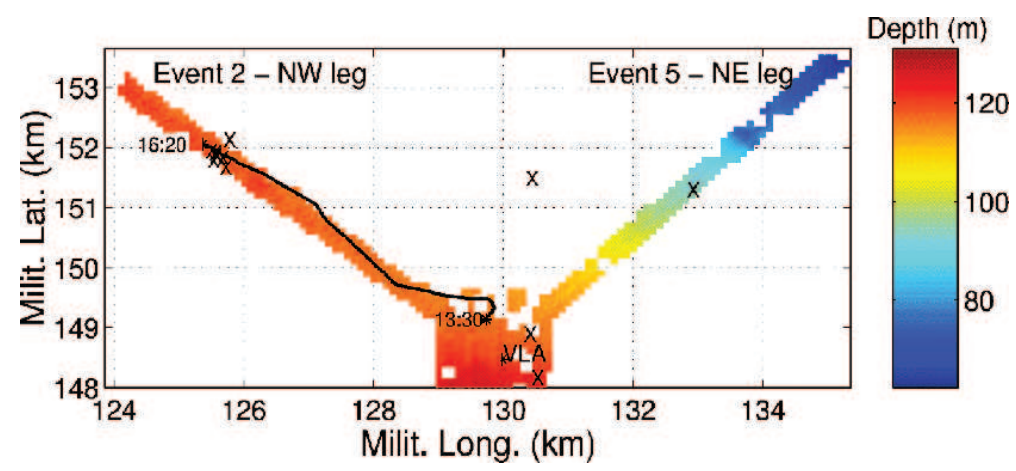

Figure 1. INTIFANTE'00 sea trial site bathymetry with XBT locations and track for Event 2.

\section{Acoustic tomography via matched-field}

\subsection{The environmental model}

For any inversion problem in underwater acoustics, the choice of an environmental model able to represent the properties of the propagation channel is an important step. That model, usually called baseline model, includes the available a priori information for the problem at hand. Since there were no in situ measurements of the seafloor properties during the experiment, a generic baseline model was chosen. It consists of a typical three-layer model with an ocean water column overlying a sediment layer and a bottom half-space, all assumed range independent, as shown in figure 2. For forward model computations the parameters were divided into subsets of water column, geometric, and geoacoustic parameters.

The water column is described by a linear combination of Empirical Orthogonal Functions (EOFs) that are constructed from representative data sampling the depth dependence of the ocean sound speed. The EOFs are obtained using singular value decomposition (SVD) of a matrix $\mathbf{C}$ with columns

$$
\underline{C}_{i}=\underline{t}_{i}-\underline{\underline{t}}
$$

where $\underline{t}_{i}$ are the real profiles available, and $\underline{\bar{t}}$ is the average profile. The SVD is known to be

$$
\mathbf{C}=\mathbf{U D V},
$$

where $\mathbf{D}$ is a diagonal matrix containing the singular values, and $\mathbf{U}$ is a matrix with orthogonal columns, which are used as the EOFs. The temperature profile is obtained by

$$
\underline{T}_{\mathrm{EOF}}=\underline{\bar{t}}+\sum_{n=1}^{N} \alpha_{n} \underline{U}_{n}
$$

where $\underline{\bar{t}}$ is the average temperature profile, $\underline{U}_{n}$ is the $\mathrm{n}^{\text {th }}$ EOF, and $N$ is the number of EOFs to be combined, judged to accurately represent the temperature profile for the problem at hand. Generally, a criteria based on the total energy contained in the first $N$ EOFs is used. The temperature profiles obtained from the XBT measurements (see $\mathbf{X}$ signs in figure 1 and profiles in figure 3(a)) served as database for the computation of the EOFs. The criteria used to select the number of relevant EOFs was

$$
\hat{N}=\min _{N}\left\{\frac{\sum_{n=1}^{N} \lambda_{n}^{2}}{\sum_{m=1}^{M} \lambda_{m}^{2}}>0.8\right\},
$$

where the $\lambda_{n}$ are the singular values obtained by the SVD, M is the total number of singular values, provided that $\lambda_{1} \geq \lambda_{2} \geq \ldots \geq \lambda_{M}$. For this data set the criteria (4) yielded $\mathrm{N}=2$, i.e., the first 


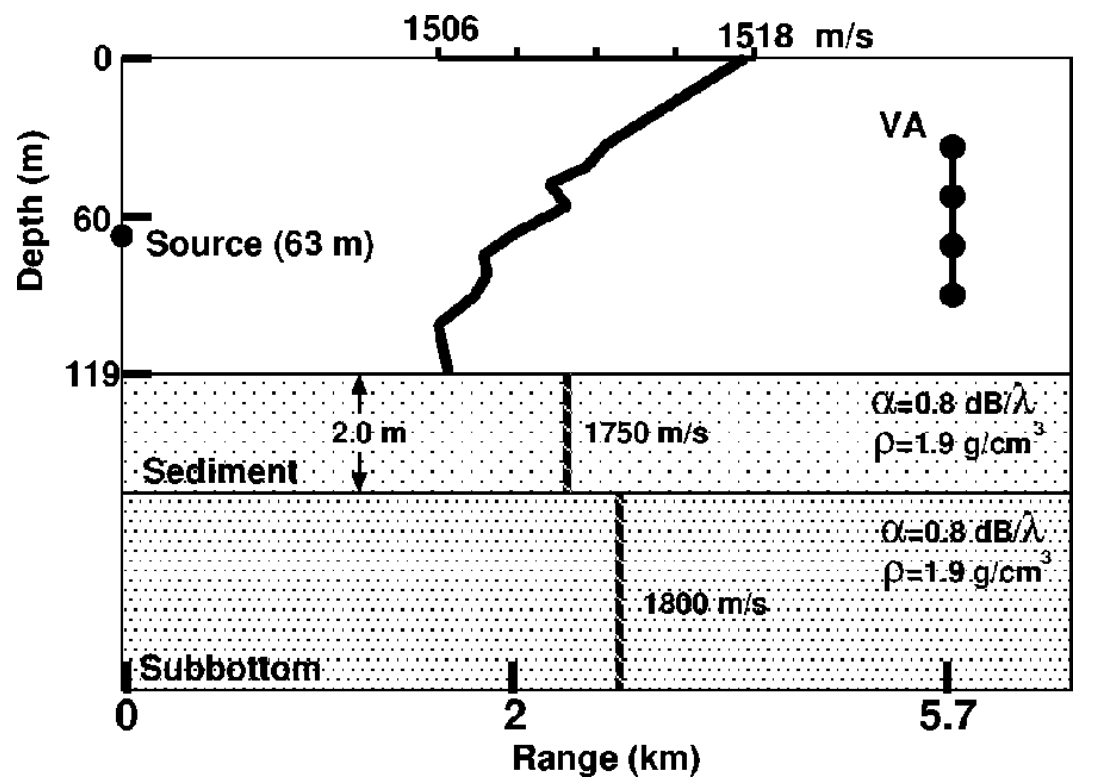

Figure 2. Baseline model for Event 2. All parameters are assumed to be range independent.

two EOFs are sufficient to model the sound speed with the requested accuracy. The coefficients $\alpha_{n}$, which are the coefficients of the linear combination of EOFs, are now part of the search space, i.e., they are searched as free parameters. The mean temperature profile and the EOFs obtained from the XBT temperature measurements are shown in figures 3(a) and 3(b).

A direct measurement of the EOF coefficients $\alpha_{n}$ can be obtained for the times at which temperature profiles were taken. This can be done by projecting those temperature profiles into the EOF subspace as

$$
\hat{\alpha}_{n}\left(t_{k}\right)=\underline{U}_{n}^{T} \underline{T}\left(t_{k}\right),
$$

where $\underline{T}\left(t_{k}\right)$ is the temperature profile measured at time $t_{k}$. Computing estimates of $\alpha_{n}$ is useful to obtain a priori information of their respective intervals of variation, and to compare with the a posteriori acoustic estimates.

The geometric subset consists of source range and depth, receiver depth, and array tilt which mainly determine the experiment geometry. The geoacoustic subset consists of the sediment soundspeed, sediment thickness and subbottom sound-speed. During the search the subbottom soundspeed $c_{b}$ was coupled to the sediment sound-speed $c_{s}$, as $c_{b}=c_{s}+\delta c_{b}$, to ensure that non-physical solutions are excluded from the search procedure. In order to test acoustic tomography with sparse populated receiver arrays, only data of every fourth hydrophone was considered in this study, resulting in a reduced acoustic aperture of $48 \mathrm{~m}$ and a receiver spacing of $16 \mathrm{~m}$.

\subsection{Data model and objective function}

Acoustic tomography can be posed as an optimization problem, that is, to find an estimate of the parameter vector $\underline{\theta}=\left[\underline{\theta}_{\alpha}^{T} \underline{\theta}_{s}^{T} \underline{\theta}_{g}^{T}\right]^{T}$ of the deterministic propagation model that best fits, according to a given objective function, the observed data. In Ref. [7] a broadband data model for the acoustic data received at an $L$-hydrophone array with the form

$$
\underline{Y}\left(\theta_{0}\right)=\left[\underline{Y}^{T}\left(\theta_{0}, \omega_{1}\right), \underline{Y}^{T}\left(\theta_{0}, \omega_{2}\right), \cdots, \underline{Y}^{T}\left(\theta_{0}, \omega_{K}\right)\right]^{T}
$$


(a)

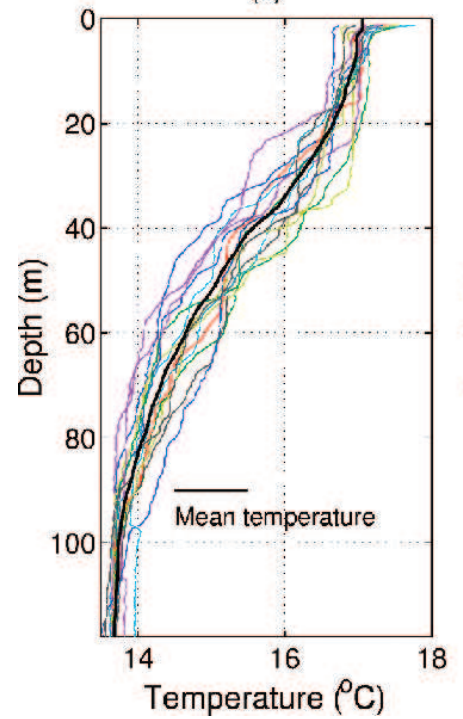

(b)

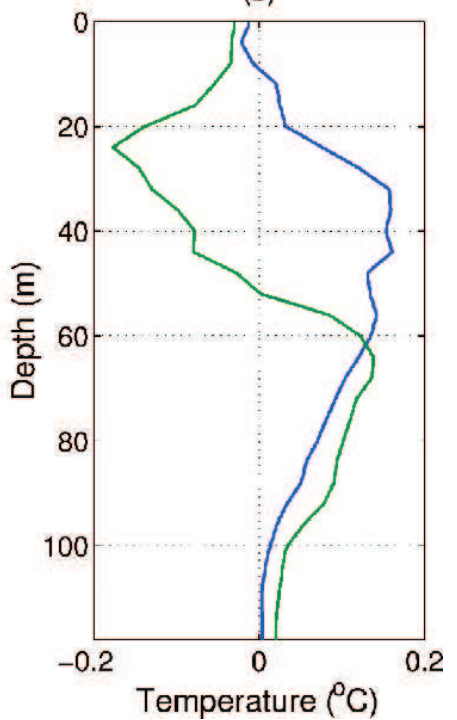

Figure 3. Temperature profiles measured during the INTIMATE'00 sea trial using XBTs, and empirical orthogonal functions obtained from the XBT temperature profiles (b).

$$
=\mathbf{H}\left(\theta_{0}\right) \underline{S}+\underline{N},
$$

was proposed in order to introduce, as much as possible, a common frame for the narrow and broadband cases. The matrix $\mathbf{H}\left(\theta_{0}\right)$ is

$$
\mathbf{H}\left(\theta_{0}\right)=\left[\begin{array}{cccc}
\underline{H}\left(\omega_{1}, \theta_{0}\right) & 0 & \ldots & 0 \\
0 & \underline{H}\left(\omega_{2}, \theta_{0}\right) & \ldots & 0 \\
\vdots & \vdots & \ddots & \vdots \\
0 & 0 & \ldots & \underline{H}\left(\omega_{K}, \theta_{0}\right)
\end{array}\right],
$$

where $K$ is the number of frequencies considered and $\underline{H}\left(\omega_{k}, \theta_{0}\right)$ is the channel response for frequency $\omega_{k}$, and the number of rows of $\mathbf{H}\left(\theta_{0}\right)$ is $K L$. $\theta_{0}$ is the parameter vector representing the relevant parameters to be estimated. The vector $\underline{S}$ has entries $S\left(\omega_{k}\right) \alpha\left(\omega_{k}\right) . S\left(\omega_{k}\right)$ is the spectrum of the emitted waveform and $\alpha\left(\omega_{k}\right)$ is a generic random perturbation factor that has been discussed in detail in Ref. [7], and was presented with the purpose of modeling small scale environmental perturbations. This follows from the observation that repeated emissions of a deterministic signal result in successive receptions suffering rapid changes, even at high SNR. The vector $\underline{N}$ represents the noise assumed independent across space and frequency, and has obviously the same notation as $\underline{Y}$ in (6).

It follows that (6) can be used directly to obtain the correlation matrix as

$$
\begin{aligned}
\mathbf{C}_{Y Y}\left(\theta_{0}\right) & =\mathrm{E}\left[\underline{\mathrm{Y}}\left(\theta_{0}\right) \underline{\mathrm{Y}}^{\mathrm{H}}\left(\theta_{0}\right)\right] \\
& =\mathbf{H}\left(\theta_{0}\right) \mathbf{C}_{S S} \mathbf{H}^{H}\left(\theta_{0}\right)+\mathbf{C}_{N N},
\end{aligned}
$$

where $\mathbf{C}_{S S}=\mathrm{E}\left[\underline{\mathrm{S}}^{\mathrm{H}}\right]$ is a signal matrix that measures the frequency cross-correlation of the received signals and has entries

$$
\left[\mathbf{C}_{S S_{i j}}=\mathrm{E}\left[\mathrm{S}\left(\omega_{\mathrm{i}}\right) \alpha\left(\omega_{\mathrm{i}}\right) \mathrm{S}^{*}\left(\omega_{\mathrm{j}}\right) \alpha^{*}\left(\omega_{\mathrm{j}}\right)\right] .\right.
$$


Matrix $\mathbf{C}_{S S}$ is diagonal if the frequency bins $S\left(\omega_{k}\right) \alpha\left(\omega_{k}\right)$ are uncorrelated across frequency. The matrix $\mathbf{C}_{N N}$ is the noise correlation matrix and is diagonal with $L \times L$ dimensional blocks $\sigma_{N}^{2}\left(\omega_{k}\right) \mathbf{I}$ if the noise is uncorrelated over space and frequency. Here it is assumed that $\sigma_{N}^{2}$ is independent of the frequency. Equation (8) has the classical form found in horizontal array beamforming where the signal matrix stands for the correlation of multiple emitters, while in the present case it stands for the correlation of multiple frequencies.

The objective function used here is the cross-frequency incoherent broadband processor obtained as

$$
P(\underline{\theta})=\frac{\operatorname{tr}\left\{\mathbf{P}_{H}(\underline{\theta}) \mathbf{C}_{Y Y} \mathbf{P}_{H}(\underline{\theta}) \mathbf{C}_{Y Y}\right\}}{\operatorname{tr}\left\{\mathbf{P}_{H}(\underline{\theta}) \mathbf{C}_{Y Y}\right\}},
$$

using the data model proposed in equation (6) and where $\operatorname{tr}\{\cdot\}$ is the matrix trace operator. Matrix $\mathbf{P}_{H}(\underline{\theta})=\mathbf{H}(\underline{\theta})\left[\mathbf{H}^{H}(\underline{\theta}) \mathbf{H}(\underline{\theta})\right]^{-1} \mathbf{H}^{H}(\underline{\theta})$ is a projection matrix, well known from topics such as subspace based, least mean squares, or maximum likelihood methods. Note that equation (10) also generates auto-frequency terms. If the signal is assumed to be uncorrelated across frequencies, and flat spectrum, i.e., $\mathbf{C}_{S S}$ is assumed identity, then a processor equivalent to the conventional incoherent Bartlett processor for $K$ frequencies is obtained:

$$
P(\underline{\theta})=\frac{\sum_{k=1}^{K} \underline{H}\left(\omega_{k}, \theta\right)^{H} \mathbf{C}_{Y Y}\left(\omega_{k}\right) \underline{H}\left(\omega_{k}, \theta\right)}{\sum_{k=1}^{K}\left\|\underline{H}\left(\omega_{k}, \theta\right)\right\|^{2}} .
$$

In practice, $\mathbf{C}_{Y Y}$, given by equation (8), is replaced by a sample correlation matrix considering $N$ snapshots, given as

$$
\hat{\mathbf{C}}_{Y Y}=\frac{1}{N} \sum_{n=1}^{N} \underline{Y}_{n}\left(\underline{\theta_{0}}\right) \underline{Y}_{n}^{H}\left(\underline{\theta_{0}}\right),
$$

with $\underline{Y}_{n}\left(\underline{\theta}_{0}\right)$ containing the $K$ data vectors $\underline{Y}_{n}\left(\underline{\theta_{0}}, \omega_{k}\right)$.

Michalopoulou [8] suggested the normalization of the received acoustic field by dividing the received signals by the signals corresponding to the hydrophone with highest SNR, in order to overcome the requirement of knowing the emitted waveform when a coherent processor is used. Observing the phases of the complex field received across the array, it can be found that a sensor coherent and frequency dependent continuous drift occurs over time. Consequently, the coherence of crossfrequency terms computed over the observation time is destroyed, causing diagonalization of the signal matrix $\mathbf{C}_{S S}$. Normalization using a reference hydrophone can also be useful to increase the coherence of the signals received along time, and reduce the diagonalization of the signal matrix $\mathbf{C}_{S S}$, and therefore the spreading of energy into different eigenvectors. This data normalization is applied during this study. However, no details on the choice of the reference hydrophone will be given here.

\subsection{Parameter focalization applied to OAT}

The present estimation problem represents a case where multiple parameters are assumed to be either unknown or known only to a certain degree. Several parameters are therefore to be optimized simultaneously in order to fit the model to the data via environmental focalization [9]. The water column temperature, parameterized by the EOF coefficients, is time variant as well as source position (both range and depth), array tilt and receiver depth. In order to reduce ambiguities, water depth is not included as search parameter but tidal variation is accounted for using a tide prediction model and a reference mean water depth of $118.7 \mathrm{~m}$. Then, adopting the spirit of the focalization procedure, it was decided to account for uncertainty in the seafloor properties, in order to improve the model fit. Table 1 shows the parameters searched, with respective search bounds and quantization steps. The search bounds reflect the use of the available a priori knowledge. For example, it was found that by projecting the XBT temperature profiles into the EOF subspace, the measured $\alpha_{n}$ are confined 
Table 1. GA forward model parameters with respective search interval. The compressional speed in the bottom is coupled to the compressional speed in the sediment.

\begin{tabular}{lccc}
\hline Model parameter & min. & max. & Quantization steps \\
\hline Temperature & & & \\
$\alpha_{1}\left({ }^{\circ} \mathrm{C}\right)$ & -5 & 5 & 32 \\
$\alpha_{2}\left({ }^{\circ} \mathrm{C}\right)$ & -5 & 5 & 32 \\
\hline Geometric & & & \\
source range $(\mathrm{km})$ & 5.4 & 6.1 & 32 \\
source depth $(\mathrm{m})$ & 70 & 85 & 32 \\
receiver depth (m) & 85 & 95 & 32 \\
tilt (rad) & -0.045 & 0.045 & 32 \\
\hline Sediment & & & \\
comp. speed (m/s) & 1520 & 1680 & 32 \\
thickness (m) & 1 & 7 & 16 \\
\hline Bottom & & & \\
comp. speed (m/s) & 1 & 200 & 32 \\
\hline
\end{tabular}

to the interval -3 and 3. However, during the data inversions a larger variation is allowed in order to avoid obtaining estimates on the boundaries of the search intervals. A priori knowledge from the experiment was used to restrict the search limits for the geometrical parameters source range and source depth.

The correlation matrices were computed considering only the time series received at 4 hydrophones, 4, 8, 12 and 16, taking 20 frequencies from 288 to $592 \mathrm{~Hz}$ with a step of $16 \mathrm{~Hz}$ obtained from 18 Fourier-transformed LFM sweeps, giving an acoustic observation time of ca. 3 minutes. This procedure was repeated with intervals not shorter than 15 minutes.

Although a great deal of a priori information is used to keep the size of the search space restricted, it is still of the order $10^{13}$. Such a huge search space can be covered using a genetic algorithm (GA), which enables a significant reduction of the number of forward models to be computed. The forward computation model used was the normal mode code C-SNAP [10] and the GA is an implementation proposed by Fassbender [11]. The population size was set to 160 and the number of iterations to 50. Three independent populations were run for each time point. The crossover probability was set to 0.7 , initial mutation probability was 0.0096 and the final mutation probability was 0.0026 , where its value was linear with the iteration number. In order to allow a more efficient search the solution at a given time point is used in the initialization of the solving procedure of the next time point. For more details see [4].

The inversion results shown in figure 4 were obtained using the conventional Bartlett processor (11). The left column contains the estimates of the $\alpha_{i}$ (black curves) together with an interpolation of the projections based on the measurements obtained with (5) - colored curves. It can be seen that the $\alpha_{i}$ are reasonably well estimated at the beginning of the run, taking one or two outliers apart, but then progressively the estimates diverge from the measurements, reaching the largest error at time 290.25. Then the $\alpha_{i}$ estimates progressively start to converge again to the measured values, where in particular $\alpha_{2}$ has some estimates coinciding with the measurements between time 290.35 at the end of the run. In the center column of figure 4, source range estimates (black curve) are of reasonable quality, with few severe errors and most estimation errors within the GPS accuracy and uncertainty caused by cable scopes in the moorings of the receiving system [12], while for the other geometric parameters some difficulties are present, specially at the beginning of the run, and around time 290.3. The geoacoustic parameters (right column) show a chaotic behavior, indicating that the seafloor properties cannot be properly retrieved from this data set. 

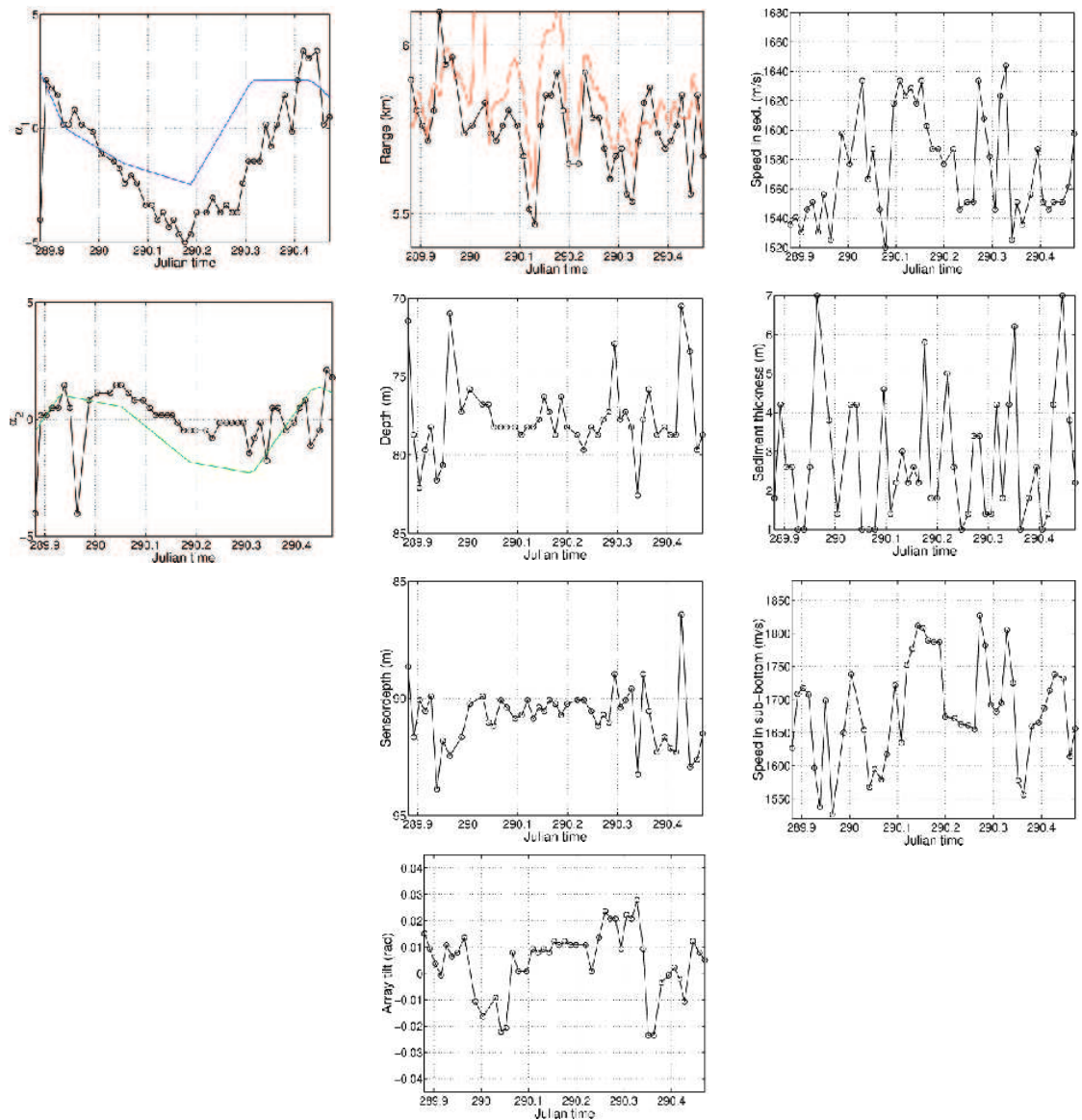

Figure 4. Focalization results obtained for Event 2.

The most important fact is that initially the inversion procedure reasonably yields the expected values for the estimative of the $\alpha_{i}$, until a progressive departure between the estimates and the measured values takes place. The main question at this point is to find one or more explanations for that occurrence. From the propagation channel point of view the question is: what has changed to cause such degradation in the quality of the estimation results? The next section attempts to find the causes for the fluctuation of the estimation performance observed above.

\section{Datamodels and eigenvalues}

Severe parameter estimation difficulties were found in part of the data analysed in the previous section. Such difficulties are potentially generated by two types of model mismatch - physical and statistical. The physical model mismatch can be originated by missing a priori environmental information about the channel and geometry, causing an erroneous or too simplistic choice of the physical model. On this side of the problem there is not much to be done since the amount of information is fixed to that available $a$ priori. The statistical model mismatch results from wrong assumptions on the statistical properties of signals received on the vertical array, and thus erroneous choice of signal 
or noise models. The problem at hand is to recall the statistical model in detail and to investigate whether any of the assumptions has been violated such that the estimation process failed to yield proper model parameter estimates.

The signal model described in (6) assumes that the field measured at the array corresponds to the channel Green's function as the solution of the wave equation at the sensor locations depending on source position and environmental parameters incorporated into a channel response vector $\underline{H}\left(\omega, \underline{\theta}_{0}\right)$. Noise perturbations are conveniently expressed in terms of covariance matrices $\mathbf{C}_{U U}$, which describe the ocean noise spatial and frequential structure. For the derivation of the matched-field processor in equation (10), it was assumed that the signals were random frequency cross-correlated and that the noise is uncorrelated across space and frequency, falling in the category of sensor noise with a diagonal structured covariance matrix.

The eigendecomposition of the covariance matrix in equation (8) can be of central importance, since the eigenvalues are a mirror of its structure and its positivity allows for the factorization in eigenvalues and eigenvectors

$$
\mathbf{C}_{Y Y}\left(\theta_{0}\right)=\mathbf{V E V}^{H},
$$

with $\mathbf{V}$ unitary and $\mathbf{E}=\operatorname{diag}\left\{e_{1}, e_{2}, \ldots, e_{K L}\right\}$ a diagonal matrix of real eigenvalues ordered such that $e_{1} \geq e_{2} \geq \ldots \geq e_{K L}>0$. In the case of uncorrelated noise, it can be observed that if the signal matrix $\mathbf{C}_{S S}$ has full rank then there will be $L(K-1)$ eigenvalues equal to $\sigma_{U}^{2}$. If, on the other hand, $\mathbf{C}_{S S}$ has rank equal 1 , then the signal component is deterministic and $L K-1$ eigenvalues are equal $\sigma_{U}^{2}$. In terms of matched-field processing this is the most convenient case, since it corresponds to matching the replica to the eigenvector associated with the largest eigenvalue of matrix $\mathbf{C}_{Y Y}$.

The ratio between the first and second eigenvalues $\left(\frac{e_{1}}{e_{2}}\right)$ is often an indicator of the adequateness of the statistical model relative to the data being processed.

In terms of eigenvalue analysis it is suitable to observe the eigenspectrum of the sample correlation matrix, in particular the behavior of the ratio between $e_{1}$ and $e_{2}$. This gives a measure of the degree of dominance of the first eigenvalue over time in order to detect changes in the structure of the data, and evaluate its disagreement relative the assumed model. The advantage of the eigenvalue analysis is that it is data-consistent, since it only depends on the received signals.

Figure 5(a) shows the eigenspectra of the sample correlation matrix versus time, with a data gap due to an interruption for changing the batteries of the VLA. Before and shortly after the data gap, the ship is moving away from the VLA location where it can be seen that the eigenspectra energy is spread out reaching significant values up to order 10/12. This is clearly due to a violation of the channel stationarity assumption for cross-covariance matrix estimation where several different channel acoustic responses were averaged together within the data window. After time 289.9 the source ship is free drifting so movements are limited to those necessary to keep the station. Surprisingly a significant energy spreading can also be noticed in the time interval 290 to 290.35 (approximately 9 hours), after which it abrupbtly falls off. Along the whole interval some spikes are also visible, which can be attributed to ship movements to maintain the station. In order to obtain a quantitative measure of the energy spreadout figure 5(b) shows the evolution of the $\frac{e_{1}}{e_{2}}$ ratio (top) and its inverse $\frac{e_{2}}{e_{1}}$ (bottom). It is clearly visible that the movement of the source ship in the first part of the run strongly reduces the $\frac{e_{1}}{e_{2}}$ ratio, that immediately increases when the ship stalls. As expected from the eigenspectra data an abrupt reduction of the $\frac{e_{1}}{e_{2}}$ ratio can also be noticed in the time interval 290 to 290.35. While it is easily understandable that the $\frac{e_{1}}{e_{2}}$ ratio remains low while the ship is moving fast, it is not so clear why this ratio remains low in the interval 290 to 290.35 during which the ship was on station and the acoustic channel responses should be relatively stable or do not suffer significant changes during the 3 min averaging window for each covariance matrix estimation. Clearly, the cause for the abrupt decrease of the $\frac{e_{1}}{e_{2}}$ ratio in that time interval is not the same as it is before the gap, neither the is the effect the same.

Thus there might be several effects causing these high variations in the spreading of the eigenspectrum. To some extent such spreading might be caused by a disagreement between the received 
(a)

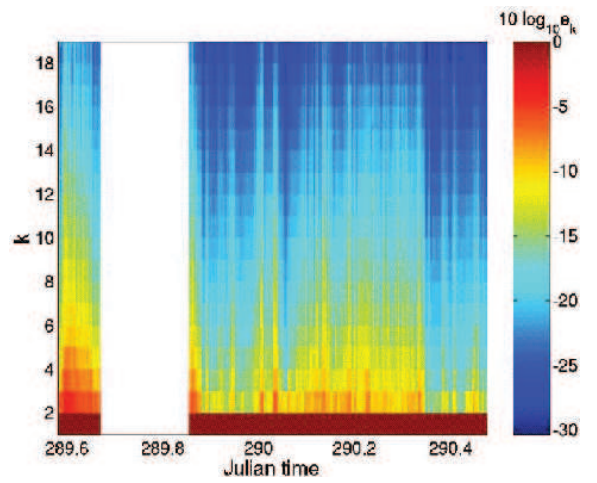

(b)
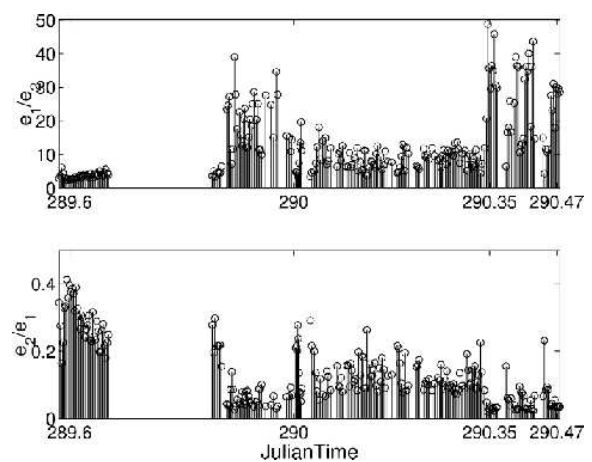

Figure 5. Eigenspectra computed from the cross-frequency correlation matrix (a); ratio between the two greatest eigenvalues $\frac{e_{1}}{e_{2}}\left(\mathrm{~b}-\right.$ top) and $\frac{e_{2}}{e_{1}}$ (b-bottom).

data and the statistical model assumed for the present problem. The rate of change in the degree of spreading at some time points configures this data set as being acquired in a highly variable scenario. The next step is to search for possible causes that can explain this model - data disagreement.

\subsection{Acoustic source motion}

The parameters concerning the source position are leading parameters in terms of acoustic field sensitivity. Thus one must account for variations in the source position during the signal emissions. In the present case the repetition rate of the emitted signal is $10 \mathrm{~s}$. The number of snapshots used to compute the sample correlation matrix is a compromise between its variance and the time coherence of the propagation channel. A number of snapshots equal to 18 represents about 3 minutes of observation time. This means that the hypothesis of a time-invariant propagation channel can possibly be violated for a given acoustic source motion. From the statistic point of view and in terms of eigenvalues, the variability of the acoustic response will induce a loss of dominance of the largest eigenvalue, due to energy partitioning in different eigenvectors. The gray line of the range vs. time plot of figure 4 reveals that the source ship was moving quite often struggling to keep the station, while being pushed towards the VLA by wind and currents. Ship motion not only implies a change on source range, but also in source depth, since, for a fixed cable scope, the source depth is a function of ship speed according to its characteristics. Thus, during accelerations, changes in the source depth occur. Figure 6(a) shows the ship speed at discrete times when acoustic data was received, and figure 6(b) shows the correlations of ship speed and ship acceleration with the $\frac{e_{2}}{e_{1}}$ ratio vs. time (figure 5 (b-bottom)). The time axis of the correlation plot is actually in time bins (for a total number of 232) since there is a gap in the data the time bins are not uniformly spaced. It can be seen that the level of $\frac{e_{2}}{e_{1}}$ is strongly correlated with the ship speed yielding a correlation above 0.9 and with the peak perfectly placed in the center of the plot, i.e., for a time lag equal to zero. The influence caused by the ship acceleration is more difficult to detect since the change in speed occurs for very short periods of time and involves a second order derivative of the position record, prone to instabilities. However, the maximum correlation still appears at the center of the function. In the present case, the source motion problem can be seen as a short time scale phenomenon. In figure 5(b) it can be clearly seen that some spikes in the $\frac{e_{2}}{e_{1}}$ ratio are standing out at several times, and those can be atributed to source motion. However, the overall energy spreading can not be accounted for by acoustic source motion only, which lead us to search for other environmental related causes. 
(a)

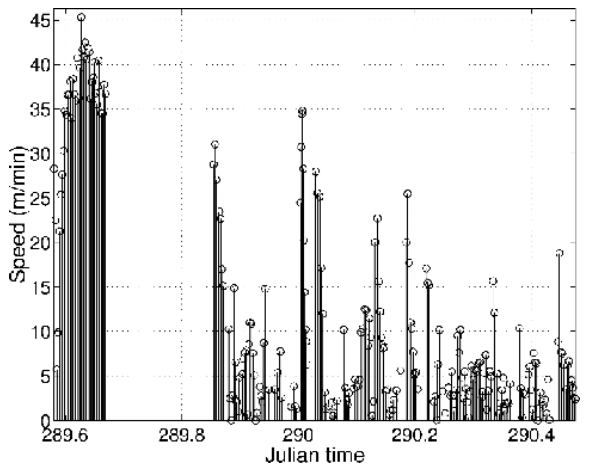

(b)

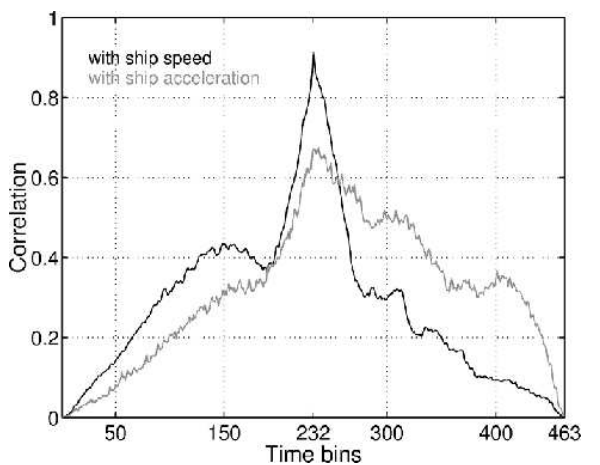

Figure 6. Correlation of ship speed and acceleration functions with the $\frac{e_{2}}{e_{1}}$ function shown in figure $5(b)$.

\subsection{Noise level and tidal forcing}

Recalling the discussion on eigenvalue analysis in the first half of section 4, it appears that the overall energy spread of the eigenspectrum in the interval 290 to 290.35 could be due to a structured noise background superimposed by short time scale variations due to source ship motion as described in the previous section.

Figure 7(a) shows a noise power estimate for hydrophone \#1 as a function of time and frequency. Here the abrupt change in noise level in the time interval 290 to 290.35 is quite clear with several strong lines across the whole frequency band, however, with some predominance below $350 \mathrm{~Hz}$. Observing the colorbar it is also found that the fluctuation of the noise level is up to $30 \mathrm{~dB}$. Finally, in figure 7(b), that shows the noise eigenspectra along time, it can be seen that the noise power abrupt changes are accompained by statistical changes denoting a correlated signal-like structure in the target interval $290-290.35$. The noise covariance matrices show strong spatial noise correlation in that period of time, which by itself is a potential reason for the breakdown of the inversion process.

It remains to find out what originated such high noise amplitude and correlation. There are several possible noise origins in the ocean. The most common is ambient noise caused by waves or wind at the ocean surface that translate in a correlated noise field at the vertical array. Other common source of correlated noise is that due to distant shipping but generally manifest itself as a variable spectrum strongly attenuated at frequencies above, say, $200 \mathrm{~Hz}$ - which obviously is not the case here. Flow noise and mechanical vibration are also common sources of noise when addressing horizontal towed arrays or vertical arrays in strong currents. Although there are no recordings of the current field during the experiment, historical records show that tidal driven currents up to $2.5 \mathrm{~m} / \mathrm{s}$ were measured in the area. Figure 7(c) shows a plot of the estimated noise level and the absolute value of the tidal forcing superimposed. The tide was predicted using a tide prediction model. It is not quite obvious that the noise level increases with the tidal forcing, but a correlation between these two quantities reveals that they are in fact strongly correlated, reaching a maximum correlation over 0.8 for zero time lag (figure 7(d)). If the current is sufficiently strong it can cause the VLA line to vibrate between the mooring and the surface buoy. In that case the vibrations can origin mechanical noise propagating along the VLA sensed by the hydrophones and resulting in spatially structured noise over a large frequency band. 
(a)

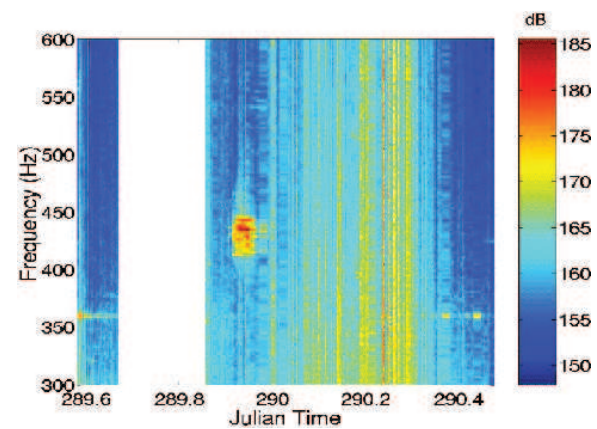

(c)

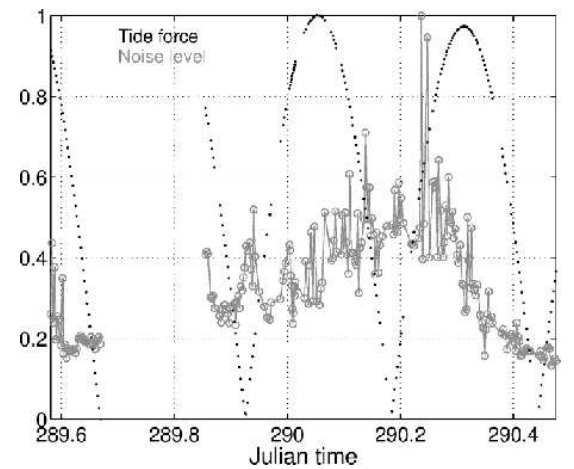

(b)

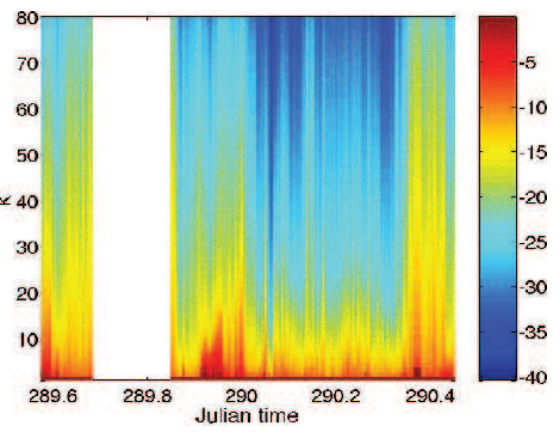

(d)

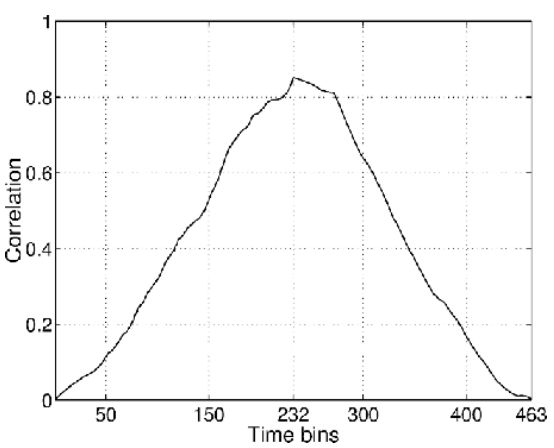

Figure 7. Estimated noise level as a function of time and frequency (a); eigenspectrum of a full rank noise covariance matrix as a function of time (b); average noise level estimated using all hydrophone with absolute tide force value over time (c); correlation of estimate noise level with absolute tide force (d).

\section{Data quality improvement for inversion}

In the sections above it was shown that various factors have a significant impact on the data structure and therefore on the inversion process. It could be seen, e.g., that if during the observation time the environment or the geometry suffers strong changes, it is likely that the energy received along time at the array tends to be spread into different eigenvectors. This is equivalent to observing a decorrelation of consecutively received signals. The duration of the observation window establishes a trade-off between the coherence of the propagation channel and the variance of the covariance matrix estimate. To handle fast varying environments one can implement an eigen-based processing technique simply based on the maximization of the $\frac{e_{1}}{e_{2}}$ ratio with the goal of adaptively determining the observation time. Ideally, this would measure the relative decorrelation rate between signals and noise.

On the other hand, high level and spatially correlated noise have shown to be an important difficulty during part of the experiment. An additional factor of difficulty is the change of the noise structure along time, where the estimated degree of cross-correlations has shown a significant variation, leading to the failure of the assumption of spatially uncorrelated noise. In that case the straight step is to gain knowledge on the noise structure and use it in the derivation of a matched-field processor assuming structured noise. Cross-frequency processors can explore the advantage of the stochastic independence of frequency bins in the frequency domain, as the result of a discrete Fourier transform 
Table 2. Summary of the four processing cases. Cross stands for cross-frequency processing; Eig. SS stands for maximization of the $\frac{e_{1}}{e_{2}}$ ratio as a function of the number of snapshots; Eig. Norm stands for maximization of the $\frac{e_{1}}{e_{2}}$ ratio as a function of normalizing the data by the best hydrophone.

\begin{tabular}{lccc}
\hline \hline & Cross & Eig. SS & Eig. Norm \\
\hline Case 1 & No & No & No \\
Case 2 & Yes & No & No \\
Case 3 & Yes & Yes & No \\
Case 4 & Yes & Yes & Yes \\
\hline \hline
\end{tabular}

property (see [7] for a complete description of the noise handling assumptions of the cross-frequency processor).

The remaining part of this section describes an attempt to improve the quality of the estimates by mitigating the difficulties discussed above. Three additional inversion results are being reported, where processing features are successively added. Four cases are summarized in table 2:

- Case 1 corresponds to the inversion reported in section 3.3 where the conventional processor is used;

- Case 2 corresponds to the application the cost function presented in equation (10), with a constant number of snapshots equal 18 and performing normalization to the best hydrophone. The idea is to take advantage of the reduced noise correlation across frequencies, since it has been observed that in part of the run extremely strong spatial noise correlation is present;

- Case 3 where the number of snapshots used for each covariance matrix is variable according to the maximization of the $\frac{e_{1}}{e_{2}}$ ratio, in order to inspect whether the variability seen during the observation window is a source of loss in parameter estimation performance, and finally

- Case 4 where the eigenvalue ratio is tested against the decision of normalization or not. The problem being addressed in this case is that of coping with drifts of the phases of the acoustic field, that prevent the field being summed coherently during the computation of the crosscorrelation matrix.

As an example of Case 3, figure 8(a) shows the result of the maximization with respect to the number of snapshots $N$ vs time. The effect of source motion at the beginning of the run is perfectly visible, both before and after the data gap. Then as the ship stalls a raise in the estimates of $N$ is observed. During the rest of the run some variability in $\hat{N}$ can be seen. Figure 8 (b) shows that the estimates of $N$ are strongly correlated with ship speed and acceleration. One interesting remark is that the correlation functions in figure 8(b) have a very similar shape of those of figure 6(b), possibly indicating that there is a close relation between the estimates of $N$ and the $\frac{e_{1}}{e_{2}}$ ratio.

The inversion results obtained along the four cases are summarized in table 3 in terms of root mean square error (RMSE) for the parameters that can be measured and are subjected to change with time, and in terms of standard deviation for the seafloor parameters that are unknown but assumed to be invariant along the whole run. Although not perfectly monotonic, it can be observed that from case 1 to case 4, an improvement can be observed for each new feature introduced in the processing. Although the improvement seems to be small, there is clearly a tendency to ameliorate the estimation quality, especially in cases 3 and 4 . The cross-frequency processor appears to perform better in conjunction with the eigen-based processing introduced in cases 3 and 4 . The introduction of the cross-frequencies in case 2 by itself has shown little or no improvement to the estimation, since the length of observation time is not addressed and the noise appears to have some frequency cross-correlation. The estimation results obtained in case 4 are not shown graphically, since, visually, it would be difficult to take striking conclusions 
(a)
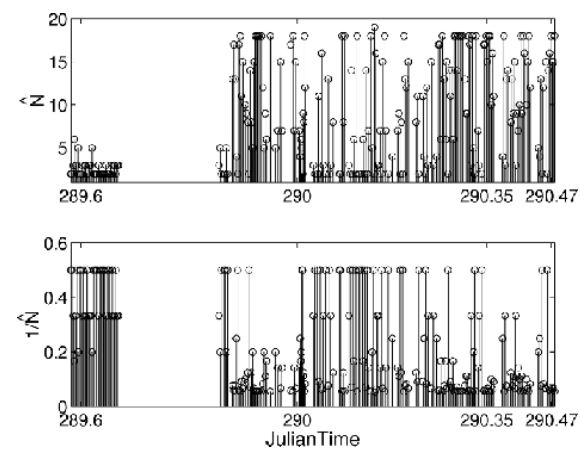

(b)

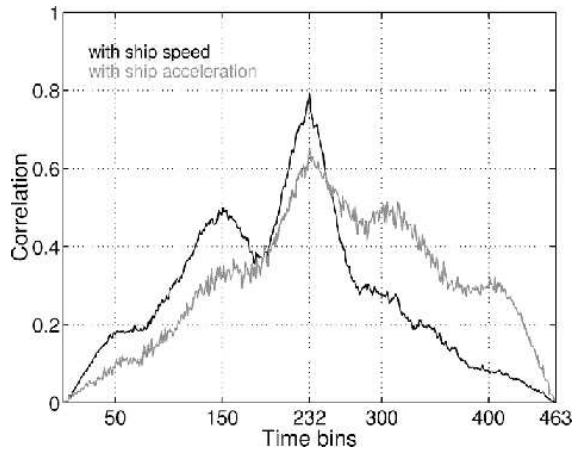

Figure 8. Estimated number of snapshot over time and its inverse (a); Correlation function of the inverse of the estimated $\mathrm{N}$ with ship speed and acceleration (b).

Table 3. Inversion performance measured via: ${ }^{1}$ RMSE using GPS source-receiver range and and XBT projected EOF coefficients; ${ }^{2}$ standard deviation of seafloor parameters (compressional velocity in sediment and subbottom, sediment thickness).

\begin{tabular}{|c|c|c|c|c|c|c|}
\hline \multirow[t]{2}{*}{ Proc. } & \multicolumn{3}{|r|}{$\mathrm{RMSE}^{1}$} & \multicolumn{3}{|c|}{$\overline{\text { Std. } \text { dev }^{2}}$} \\
\hline & $\begin{array}{c}R_{s} \\
(\mathrm{~km})\end{array}$ & $\begin{array}{c}\alpha_{1} \\
\left({ }^{\circ} \mathrm{C}\right)\end{array}$ & $\begin{array}{c}\alpha_{2} \\
\left({ }^{\circ} \mathrm{C}\right)\end{array}$ & $\begin{array}{c}c_{s} \\
(\mathrm{~m} / \mathrm{s})\end{array}$ & $\begin{array}{c}h \\
(\mathrm{~m})\end{array}$ & $\begin{array}{c}c_{b} \\
(\mathrm{~m} / \mathrm{s})\end{array}$ \\
\hline case 1 & 0.125 & 2.289 & 1.458 & 36 & 1.65 & 78 \\
\hline case 2 & 0.126 & 2.283 & 1.253 & 38 & 1.48 & 80 \\
\hline case 3 & 0.121 & 2.130 & 1.161 & 35 & 1.45 & 69 \\
\hline case 4 & 0.117 & 2.055 & 1.204 & 28 & 1.40 & 62 \\
\hline
\end{tabular}

\section{Conclusion}

In scenarios where a great deal of information is available, matched-field methods are highly reliable. Thus, they are suitable to be applied to OAT, where the number of unknown parameters is potentially high.

The INTIFANTE sea trial, conducted in the vicinity of Setúbal in October 2000, served among others, the purpose of acquiring data for testing OAT at ranges up to $6 \mathrm{~km}$ in a range-independent shallow water environment. When compared to other more complex scenarios this appeared to be a relatively easy task for MFT inversion of water column properties. Indeed during part of the run the inversion of the water temperature profile provided consistent results. However, in a relatively benign portion where the source ship was maintaining a station at $5.8 \mathrm{~km}$ distance from the VLA, the estimations significantly departed from the expected results. The search for the causes of this intriguing performance was the motivation of this paper that took us through various subjects such as the influence of rapid source motion within the limits of the stationarity assumption and the role of highly correlated noise possibly due to tidal flow at the array site. During the study the problem was identified but its origin was not completely understood.

Several processing schemes were implemented in an attempt to compensate these influences. First it was supposed that the overall quality of the data entering the inversion could be ameliorated by using cross-frequency covariance matrices. This resulted in little improvement, since the strong spatial noise correlation extended to the cross-frequency data as well. Then, in two additional attempts an eigenvalue approach was tested in order to improve the data quality. It was shown that energy partitioning into different eigenvectors was caused by source motion during the observation 
time defined a priori. This was mitigated by optimizing the $\frac{e_{1}}{e_{2}}$ ratio for observation time and data normalization. It could be shown that the estimator for the best number of snapshots was highly correlated with the ship speed and acceleration. The inversion results could be progressively improved remaining, however, strongly limited by the high noise level. Further work is necessary to treat this problem should be treated in terms of spatial filtering by making use of a prior estimated noise structure.

\section{References}

1. Munk W., Worcester P. and Wunsch C. Ocean acoustic tomography. Monographs on mechanics. University Press, Cambridge, (1995).

2. Tolstoy A. and Diachok O., Acoustic tomography via matched field processing. J. Acoust. Soc. Am. 89(3), 1119-1127 (1991).

3. Jesus S. M., Coelho E., Onofre J., Picco P., Soares C. and Lopes C., The intifante'00 sea trial: preliminary source localization and ocean tomography data analysis. In Proc. of the MTS/IEEE Oceans 2001, Hawaii, USA, November (2001).

4. Jesus S. M., Soares C., Onofre J. and Picco P., Blind ocean acoustic tomography: experimental results on the intifante'00 data set. In Proc. of the European Conference of Underwater Acoustics, Gdansk, Poland, June (2002).

5. Jesus S. M., Soares C., Onofre J., Coelho E. and Picco P., Experimental testing of the blind ocean acoustic tomography concept. In Impact of Littoral Environmental Variability on Acoustic Predictions and Sonar Performance, Lerici, Italy, September (2002).

6. Jesus S. M. and Soares C., Blind ocean acoustic tomography with source spectrum estimation. In Proceedings International Conference on Theoretical and Computational Acoustics, Hawaii, USA, August (2003).

7. Soares C. and Jesus S. M., Broadband matched field processing: Coherent and incoherent approaches. J. Acoust. Soc. Am. 113(5), 2587-2598 May (2003).

8. Michalopoulou Z.-H., Source tracking in the Hudson Canyon experiment. J. Comput. Acoust. 4, 371-383 (1996).

9. Collins M. D. and Kuperman W. A., Focalization: Environmental focusing and source localization. J. Acoust. Soc. America 90, 1410-1422 (1991).

10. Ferla C. M., Porter M. B. and Jensen F. B., C-SNAP: Coupled SACLANTCEN normal mode propagation loss model. Memorandum SM-274, SACLANTCEN Undersea Research Center, La Spezia, Italy (1993).

11. Fassbender T., Erweiterte genetische algorithmen zur globalen optimierung multimodaler funktionen. Diplomarbeit, Ruhr-Universität, Bochum, (1995).

12. Felisberto P., Lopes C. and Jesus S. M., An autonomous system for ocean acoustic tomography Sea Technology, 45(4), 17-23 (2004). 